\title{
Antimicrobial sensitivity pattern of Salmonella typhi isolated from blood culture in a referral hospital
}

\author{
Bulbul Hasan ${ }^{1}$ Sabera Gul Nahar ${ }^{1}$ Laila Akter ${ }^{2}$ Ahmed Abu Saleh ${ }^{3}$ \\ ${ }^{1}$ Department of Microbiology, Rajshahi Medical College, Rajshahi, ${ }^{2}$ Department of Microbiology, Islami Bank Medical College, \\ Rajshahi; ${ }^{3}$ Department of Microbiology \& Immunology, Bangabandhu Sheikh Mujib Medical University, Dhaka.
}

\begin{abstract}
The present study has been carried out in an attempt to evaluate antimicrobial susceptibility patterns with special reference to susceptibility of Salmonella Typhi to ciprofloxacin isolated from blood culture. The study is also designed to find out the MIC of Ciprofloxacin by E- test. Blood samples were taken for culture sensitivity, Widal test and ICT from 100 clinically suspected cases of typhoid fever in $1^{\text {st }}$ week of illness who attended at out patient department of Rajshahi Medical College Hospital (RMCH).The study was done in Microbiology Department of Rajshahi Medical College and Shishu Hospital, Dhaka. Diagnosis of patients was based on history of fever, blood culture, Widal test and ICT. The antimicrobial susceptibility pattern of isolates from blood culture was recorded. Further more, the minimum inhibitory concentration of Ciprofloxacin was determined by E-test for the isolates resistance to Ciprofloxacin. Out of 100 suspected cases of typhoid fever, blood culture positive for $S$. Typhi were 16 (16\%). Antimicrobial susceptibility pattern of 16 isolates of $S$. Typhi showed that no isolate was resistant to Ceftriaxone and Ceftazidime, only 03(18.75\%) were resistant to Ciprofloxacin and Azithromycin whereas 10(62.5\%) were MDR showing resistance to Ampicillin, Co-trimoxazole and Chloramphenicol which are first-line antityphoidal drugs. On the other hand, all $(\mathbf{1 0 0 \% )})$ the isolates were resistant to Nalidixic acid.The study revealed that Ceftriaxone and Ceftazidime are the most effective drugs in the treatment of typhoid fever. Moreover, E-test has been found to be helpful to determine appropriate therapeutic dose of Ciprofloxacin specially in case of drug resistance and pediatric population.
\end{abstract}

Key Words: Antimicrobial susceptibility, Salmonella typhi, Typhoid fever, Blood culture, Multidrug resistance.

\section{Introduction}

Typhoid fever is endemic in many developing countries particularly in the Indian subcontinent including Bangladesh ${ }^{1,2}$. In India, it is endemic with morbidity ranging from 102 to 2219 per 100,000 populations ${ }^{3}$. Current estimates from the World Health Organization (WHO) suggest that there are 16.6 million cases of typhoid fever with 600,000 deaths annually 4 . Today due to its changing modes of presentation, as well as the development of multidrug resistance, typhoid fever is becoming increasingly difficult to diagnose and treat.

\section{Correspondence:}

Dr. Md. Bulbul Hasan

Assistant Professor

Department of Microbiology,

Rajshahi Medical College,

Rajshahi-6000, Bangladesh.

Mobile no.:01740980319

E-mail: bulbul010163@yahoo.com
Improved standards of public health have resulted in a marked decline in the incidence of typhoid fever in developed countries ${ }^{5}$. The emergence of strains of Salmonella typhi resistant to multiple antibiotics poses a serious problem. The first major epidemic of multidrug resistant $S$. Typhi was reported in 1972 in Mexico ${ }^{6}$. Since then, an increasing frequency of antibiotic resistance has been reported from all parts of the world, but more so from the developing countries $^{7}$. The uses of chloramphenicol, ampicillin and cotrimoxazole have become infrequent and quinolones have become the first line of treatment of typhoid fever. However, over the last few years there has been increase in the defervescence period in patients treated with quinolones. Hence, this study is undertaken to evaluate the antibiotic response in typhoid fever. According to a recently-revised global estimate; it causes 21.6 million illnesses every year, 
resulting in 216,500 deaths $^{8}$.

Multidrug-resistant S. typhi (MDRST) is epidemiologically defined as strains resistant to any two antimicrobials in vitro even if the antimicrobials tested are known to be clinically ineffective. ${ }^{9}$ A more useful definition of MDRST is reserved for strains resistant to all three first-line antityphoidal antimicrobial agents, namely ampicillin, chloramphenicol, and trimethoprim-sulphamethoxazole ${ }^{9}$.

Typhoid fever, caused by MDRST, has become a significant cause of morbidity and mortality over recent years. With the emergence of MDRST, fluoroquinolones have gained importance for the treatment of enteric fever in recent years.

A considerable variation has been noted in the antimicrobial susceptibility patterns among isolates of $S$. Typhi as suggested in various studies conducted in different geographical locations ${ }^{10}$. Knowledge of the prevalence of $S$. Typhi and their antimicrobial susceptibility patterns is of utmost importance in the institution of appropriate antimicrobial therapy.

The estimated mean incidence of typhoid fever is 150 and 900 (per 100000 people per annum) in South America and Asia. There are an estimated 13 million cases of typhoid fever per year in Asia ${ }^{11}$.

Early implication of appropriate antimicrobial therapy requires prompt identification of the causative agent in any infectious disease including typhoid fever. Recently, continued dependence on Ciprofloxacin for the empirical treatment of typhoid fever in many developing countries including Bangladesh has lead to emergence of resistance of Salmonella Typhi to this drug. So antibiotic susceptibility test (AST) has an important role in the treatment of typhoid fever. Although the conventional method of antibiotic susceptibility testing by disk diffusion method is used to select appropriate antimicrobial drug but determination of minimal inhibitory concentration (MIC) of suitable antibiotic by recently introduced E-test can be of great help to estimate the proper dose especially for pediatric population ${ }^{12,13}$. The study is also designed to find out the MIC of Ciprofloxacin by E- test.

\section{METHODOLOGY}

\section{Study population}

One hundred (100) clinically suspected cases of typhoid fever of different age and sex attended at out patient department of Rajshahi Medical College Hospital (RMCH) from July 2006 to June 2007 were included in this study. The patients were selected according to clinical features which include fever, chills, rigor, altered bowel habit, rose spot on the trunk, bradycardia, headache, myalgia etc. and having fever for more than 7 days were considered as typhoid suspects. Blood samples were taken for culture sensitivity, Widal test and ICT from 100 clinically suspected cases of typhoid fever. Trypticase soya broth which establishes the growth of all common pathogens causing bacteraemia/ septicaemia was used as a culture medium. Collection of blood, incubation, and subculture onto MacConkey agar were done as per the standard methods ${ }^{14}$.

Suspected non-lactose-fermenting colonies were further processed and identified by biochemical reactions and confirmed by group and type-specific Salmonella antisera (Group D factor 9).

All the isolates of Salmonella Typhi were tested for their antimicrobial susceptibility pattern on Mueller Hinton agar media by disc diffusion method against Ampicillin (10 $\mu \mathrm{gm})$, Ciprofloxacin $(5 \mu \mathrm{gm})$, Cotrimoxazole $(25 \mu \mathrm{gm})$, Ceftriaxone (30 $\mu \mathrm{gm})$, Azithromycin (15 $\mu \mathrm{gm})$, Chloramphenicol (30 $\mu \mathrm{gm})$, Ceftazidim (30 $\mu \mathrm{gm})$ and Nalidixic acid (30 $\mu \mathrm{gm})$. The disk strength and zone-size interpretation was in accordance with the National Committee for Clinical Laboratory Standards (NCCLS).

\section{Interpretation of zone size}

Inhibition zones produced by each drug was considered into two susceptibility categories namely sensitive (S) and Resistant (R) shown in table-I (NCCLS, 1988). Strains produce zone size between sensitive and resistant are regarded as intermediate sensitive which is not considered in this study.

Table-I: Interpretation of zone of inhibition (NCCLS, 1988)

\begin{tabular}{lccc}
\hline Antibiotics & Disc potency & \multicolumn{2}{c}{ Diameter of zone of inhibition } \\
& $\boldsymbol{\mu g}$ /disc & Resistant $<$ mm (R) & Sensitive $>$ mm (S) \\
\hline Ampicillin & 10 & 13 & 17 \\
Chloramphenicol & 30 & 12 & 18 \\
Cotrimoxazole & 25 & 10 & 16 \\
Ciprofloxacin & 05 & 15 & 21 \\
Ceftriaxone & 30 & 13 & 21 \\
Ceftizidime & 30 & 14 & 18 \\
Nalidixic acid & 30 & 13 & 19 \\
Azithromycin & 15 & 13 & 18 \\
\hline
\end{tabular}


Furthermore; the minimum inhibitory concentration of Ciprofloxacin was determined by E-test for the isolates of $S$. Typhi resistance to Ciprofloxacin. E-test was performed on Mueller Hinton agar media. Epsilometer has been developed for a direct quantification of antimicrobial susceptibility of $S$. Typhi. A predefined continuous and exponential gradient of Ciprofloxacin concentration is immobilized along a plastic test strip. The test strip was applied to the surface of the freshly inoculated agar plate. After over night incubation a tear-drop-shaped (elliptical) zone of inhibition was seen. The zone edge intersects the graded test strip at the minimum inhibitory concentration of Ciprofloxacin.

\section{Results}

A total of 100 clinically suspected cases of typhoid fever were studied. Study population was from all age groups. Among the suspected cases 58 were male and 42 were female; male to female ratio is 1.37: 1 (Table-II).

Table-II: Sex distribution of the patients $(\mathrm{N}=100)$

\begin{tabular}{lcc}
\hline Sex & No. of patients & Ratio \\
\hline Male & 58 & 1.37 \\
Female & 42 & 1 \\
\hline
\end{tabular}

Out of 100 suspected cases of typhoid fever, blood culture positive for $S$. Typhi were $16(16 \%)$ and remaining $84(84 \%)$ were negative (Figure - I). Study population were divided into two different age groups viz., up to 15 years of age were categorized as pediatric group and above 15 years as adult group. The rate of isolation of $S$. Typhi was noted higher (17.54\%) among pediatric group when compared to adult group (13.95\%), which is shown in Table-III.

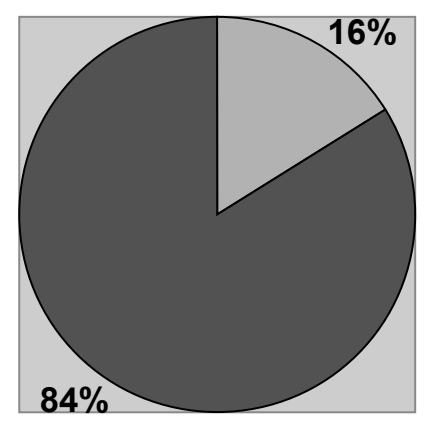

$\square$ Culture positive

cases

$\square$ Culture negative

cases

Fig - I: Rate of isolation of S. Typhi in blood culture
Table-III: Rate of isolation of $S$. Typhi in relation to age groups

\begin{tabular}{lcc}
\hline Age groups & $\begin{array}{c}\text { No. of } \\
\text { suspected cases }\end{array}$ & No. of isolates \\
\hline $\begin{array}{l}\text { Pediatric group } \\
\text { (Up to 15 years) }\end{array}$ & $57(57)^{*}$ & $10(17.54)^{*}$ \\
$\begin{array}{l}\text { Adult group } \\
\text { (Above 15 years) }\end{array}$ & $43(43)^{*}$ & $6(13.95)^{*}$ \\
Total & 100 & 16 \\
\hline
\end{tabular}

*Figures within parenthesis indicate percentage

Antimicrobial susceptibility pattern of 16 isolates of $S$. Typhi show that no isolate was resistant to Ceftriaxone and Ceftazidim, only 03(18.75\%) were resistant to Ciprofloxacin and Azithromycin whereas 10(62.5\%) were MDR showing resistance to Ampicillin, Co-trimoxazole and Chloramphenicol which are first-line antityphoidal drugs. On the other hand, all $(100 \%)$ the isolates were resistant to Nalidixic acid (Table- IV).

Table - IV: Antimicrobial Susceptibility pattern of $S$. Typhi ( $\mathrm{n}=16)$

\begin{tabular}{lcc}
\hline Antimicrobial agent & Susceptibility pattern & S. Typhi \\
\hline Ceftriaxone & S & $16(100)$ \\
Ceftazidime & $\mathrm{R}$ & 00 \\
Ciprofloxacin & $\mathrm{S}$ & $16(100)$ \\
& $\mathrm{R}$ & 00 \\
Azithromycin & $\mathrm{S}$ & $13(81.25)$ \\
& $\mathrm{R}$ & $03(18.75)$ \\
Ampicillin & $\mathrm{S}$ & $13(81.25)$ \\
& $\mathrm{R}$ & $03(18.75)$ \\
Cotrimoxazole & $\mathrm{S}$ & $06(37.50)$ \\
& $\mathrm{R}$ & $10(62.50)$ \\
Cloramphenicol & $\mathrm{S}$ & $06(37.50)$ \\
& $\mathrm{R}$ & $10(62.50)$ \\
Nalidixic Acid & $\mathrm{S}$ & $06(37.50)$ \\
\hline Figures within parenthesis indicate percentage. & $10(62.50)$ \\
R: Resistant & $\mathrm{R}$ & 00 \\
Sensitive & $\mathrm{S}$ & $16(100)$ \\
\hline & & \\
& $\mathrm{R}$ & \\
& &
\end{tabular}


The zone of inhibition of Ciprofloxacin in disc diffusion method was $10 \mathrm{~mm}$ and the minimum inhibitory concentration of Ciprofloxacin by E-strip was $24 \mu \mathrm{g} / \mathrm{ml}$ (Figure-II).

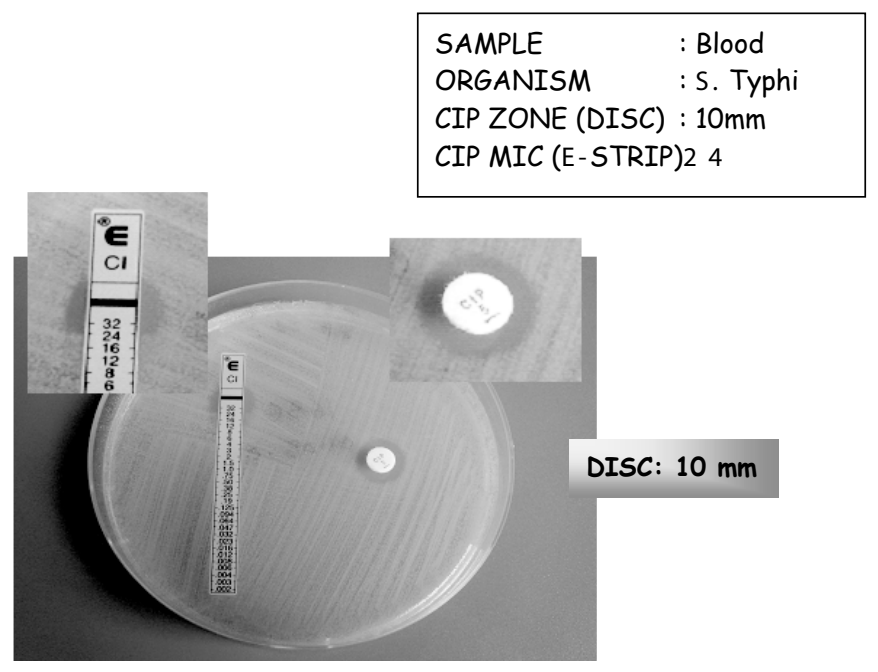

MIC of Ciprofloxacin in E-strip

Figure-II Determination of MIC for Cipro-Resistant Salmonella Typhi

\section{Discussion}

Enteric fever is still a significant public health problem in many developing countries. It is a dreaded disease because of its long course and associated complications if not detected and treated early. There are reports of changing clinical features in typhoid fever caused by drug resistant $S$. Typhi leading to difficulty in clinical diagnosis ${ }^{15,16}$.

Drug resistance in typhoid fever is considered as one of the important factors in the morbidity and mortality of the disease. Since the introduction of chloramphenicol in 1948, it has been the drug of choice in the treatment of typhoid fever in most parts of the world. But indiscriminate use of the drug and acquisition of plasmid mediated $\mathrm{R}$ factor has led to the development of resistance to $S$. Typhi against this drug ${ }^{17}$.

Typhoid fever is endemic in Bangladesh, where there is a high incidence in children ${ }^{18}$. The emergence of MDR $S$. Typhi isolates in the early 1990s, particularly from the Indian subcontinent, prompted the suggestion that ceftriaxone, ceftazidim and ciprofloxacin should be the drug of choice for empirical treatment of typhoid fever ${ }^{19,20,21}$. Initially, reduced use of amoxicillin, cotrimoxazole, or chloramphenicol was associated with a decreased prevalence of MDR strains, but more recently, continued dependence on ciprofloxacin for the empirical treatment of typhoid fever in Bangladesh and elsewhere has led to the emergence of resistance of $S$. Typhi to this drug 22,23 .

In this context of changing the dynamics of resistance to antibiotics, it is imperative for optimal patient care that accurate and early isolation of $S$. Typhi and its antibiotic susceptibility pattern be available to the clinician. Although the conventional method of antibiotic susceptibility testing by disc diffusion method is used to select appropriate antimicrobial drug but determination of minimum inhibitory concentration (MIC) of suitable antibiotic by recently introduced E-test can be of great help to estimate the proper therapeutic dose specially for pediatric population and drug resistant situation ${ }^{12,13}$. In a Ciprofloxacin resistant case the zone of inhibition was $10 \mathrm{~mm}$ in disk diffusion method and the minimum inhibitory concentration by E-strip was found $24 \mu \mathrm{gm} / \mathrm{ml}$ in the present study.

\section{Conclusion}

The public health burden of typhoid fever can be substantially reduced by rapid diagnosis and appropriate antibiotic therapy. The present study revealed that Ceftriaxone and Ceftazidim are the most effective drug against $S$. Typhi. Although the role of Ciprofloxacin in the treatment of typhoid fever has recently been made controversial among the clinicians but the efficacy of Ciprofloxacin in the present study has been found to be more than $80 \%$. So, more work need to be carried out to evaluate the status of Ciprofloxacin.

This study also helps the physician to calculate the proper therapeutic dose of Ciprofloxacin by E-test and thus minimize drug resistance. E-test can be done to determine the appropriate therapeutic dose of commonly used antibiotics in typhoid fever in case of drug resistance or pediatric population.

This approach may improve the quality and cost of patient care in the developing world, where typhoid fever is endemic, MDR strains are relatively common, and the availability of advanced diagnostic laboratory methods is limited. 


\section{Reference}

1. Rockhill RC, Lesmana M, Moechtar MA, Sutomo A. Detection of Salmonella Ci, D and Vi antigens by Coagglutination in blood culture from patients with Salmonella infections. Southeast Asian J Trop Med Publ HLTH 1980; 11: 441-445.

2. Saha SK, Amin, Hanif M, Islam M \& Khan WA. Interpretation of the Widal test in the diagnosis of typhoid fever in Bangladeshi children. Annals of Tropical Paediatrics 1996; 16: 75-78.

3. Mehta PJ, Hakim A, Kamath S. The changing faces of salmonellosis. J Assoc Physicians India 1992:40;713.

4. White NJ, Parry CM. The treatment of typhoid fever. Current opinion Infect Disease 1996; 9: 298-302.

5. Gulati PD, Saxena SN, Gupta PS, Chuttani HK. Changing pattern of typhoid fever. Am J Med 1968;45;544-8.

6. Edelman R, Levine MM. Summary of an international workshop on typhoid fever. Rev Infect Dis 1986;8:32949.

7. Samantray SK. Typhoid fever resistant to furazolidine, Ampicillin, chloramphenicol and co-trimoxazole. Indian J Med Sci 1979; 33 ;1-3.

8. Crump JA, Luby SP, Mintz ED. The global burden of typhoid fever. Bull World Health Organ. 2004;82:346-53.

9. Le TP, Hoffman SL. Typhoid fever. In: Guerrant RL, Walker DH, Weller PF, editors. Tropical infectious diseases: principles, pathogens and practice. Philadelphia, PA: Livingstone; 1999. pp. 277-95.

10. Rahman M, Ahmad A, Shoma S. Decline in epidemic of multidrug resistant Salmonella typhi is not associated with increased incidence of antibiotic-susceptible strain in Bangladesh. Epidemiol Infect. 2002;129:29-34.

11. Ivanoff B, Levin MM, Lambert PH. Vaccination against typhoid fever: Present status. Bull WHO 1994; 72: (6) 957-971.
12. Bauer AWKirby WMM SherrisJC and Ture M. Antimicrobial susceptibility testing by a standardized single disk method. Am J Clin Pathol 1966; 45:493-496.

13. Andrews J M ,Bradley J E, Wise R. Comparison of "E" test with conventional agar MIC.1993 Journal of Antimicrobial Chemotherapy31; 802-803.

14. Collee JG, Miles RS, Watt B. Tests for the identification of bacteria. In: Collee JG, Fraser AG, Marmion BP, Simmons A, editors. Mackie and McCartney Practical medical microbiology, 14th ed. London: Livingstone; 1996. pp. 131-49.

15. Bhutta ZA, Nagvi SH, Razzaq RA, Farooqui BJ. Multidrug resistant typhoid in children : Presentation and Clinical features. Rev Infec Dis 1991; 13: $832-836$.

16. Butta ZA. Impact of age and drug resistance on mortality in typhoid fever. Arch Dis Chi 1996; 75: 214-217.

17. Agarwal KC, PanHotra BR, Mahanta J. Typhoid fever due to chloramphenicol resistant S. typhi associated with ' $r$ ' plasmid. Indian J Med Res 1981;73:484-8.

18. Saha S K, Baqui A H, Hanif M, et al. Typhoid fever in Bangladesh: implications for vaccination policy. Pediatr Infect Dis J. 2001;20:521-524.

19. Jesudassan M V, Jacob J T. Multiresistant Salmonella typhi in India. Lancet. 1990;336:252.

20. Saha S K, Saha S. Antibiotic resistance of Salmonella typhi in Bangladesh. J Antimicrob Chemother. 1994; 33:190-191.

21. Hasan Bulbul. Study on the Laboratory Diagnosis and Drug Resistance in Typhoid Fever [M. Phil (Microbiology) Thesis], RMC. 2007; 83.

22. Murdoch D A, Banatvaia N, Bone A, Shoismatulloev B I, Ward L R, Threlfall E J. Epidemic ciprofloxacin-resistant Salmonella typhi in Tajakistan. Lancet. 1998;351:339.

23. Saha S K, Talukder S Y, Islam M, Saha S. A highly cefriaxone-resistant Salmonella typhi in Bangladesh. Pediatr Infect Dis J. 1999;18:387. 